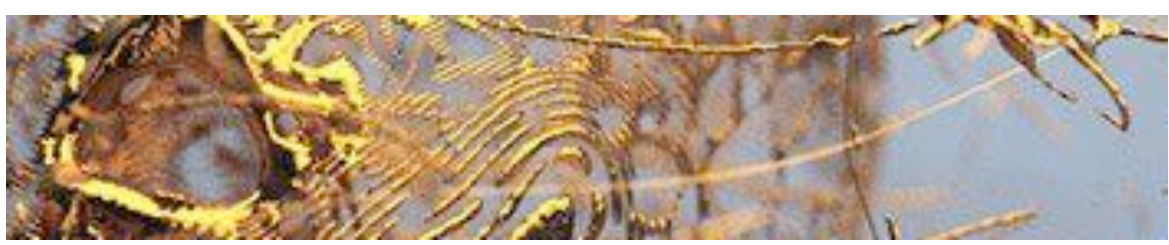

\title{
La producción de programas dramatizados radiales: un análisis desde las mediaciones.
}

\section{The production of dramatized radio programs: an analysis from the mediations.}

\author{
Beatriz Elena Fonseca Muñoz ${ }^{(*)}$ \\ Universidad de la Habana- Cuba \\ beatriselena@,fcom.uh.cu
}

\begin{abstract}
Resumen
Muchas son las generaciones que han crecido, soñado, sufrido, reido, entre otras experiencias, con la magia que transmiten los mensajes radiales. No obstante, en términos de investigación, el medio radial en la realidad cubana no ha tenido una fuerte presencia. En esta última década se observa un discreto interés entre los investigadores por el estudio de este medio. Los programas dramatizados radiales son unos de los menos investigados y es por ello que en el presente trabajo se destaca el momento de su producción, analizándolo desde la teoría de las mediaciones de Manuel Martín Serrano. Esto posibilita examinar el comporta-
\end{abstract}

\begin{abstract}
Many are the generations that have grown, dreamt, suffered, laughed, among many other experiences, with the magic transmitted by radio messages. However, on terms of research, the radio media on the Cuban reality has not had a strong presence. In this last decade, a discrete interest is observed among researchers for the study of this media. The dramatized radio programs are one of the less researched and that's why the present job the highlights the moment of its production, analyzing it from the theory of mediations from Manuel Martín Serrano. This enables to examine the behavior of this process in a determined historical
\end{abstract}


miento de este proceso en un contexto histórico determinado.

Palabras clave: Radio cubana, dramatizados radiales, Radioarte, mediaciones, producción de programas. context.

Keywords: Cuban Radio, dramatized radio, Radioarte, mediations, production of programs.

\section{INTRODUCCIÓN}

Sistematizado por Martín Serrano (1993), el enfoque de los estudios en comunicación desde las mediaciones permite el análisis de las mismas en los diferentes momentos del proceso comunicativo, si bien es cierto que el de mayor interés ha sido la recepción, en la cual marcan pautas las investigaciones de autores como Martín Barbero (1987).

El objetivo del presente trabajo es examinar el proceso de producción de los dramatizados radiales en el contexto cubano, específicamente en la productora Radioarte, desde el enfoque de las mediaciones, pues "La elaboración de un método para el estudio de la producción social de comunicación permite comprender cómo intervienen las instituciones mediadoras con sus productos comunicativos en la propuesta de visiones del mundo" (Martín Serrano, 1993: 106).

El medio radial resulta muy poco favorecido en el campo investigativo, más aún desde las posiciones del emisor y sus modos de hacer. Según estudios realizados ${ }^{1}$ la investigación sobre la radio ha abarcado las siguientes temáticas, en sentido general:

- Estudios históricos de algunas emisoras provinciales y municipales o de determinados programas no dramatizados.

- Análisis de contenido de programas no dramatizados, fundamentalmente informativos.

- Análisis de contenido de una programación con un perfil determinado. Por ejemplo, la infantil y juvenil.

1 Nos referimos a los estudios realizados por los miembros de la Línea de Investigación Campo Académico de la Comunicación en Cuba, coordinada por la Facultad de Comunicación de la Universidad de La Habana en vínculo con otras instituciones docentes y científicas del país. 
- Valoración de la calidad de diferentes programas no dramatizados.

- Investigación de audiencia.

Los resultados anteriores demuestran que los emisores apenas han sido objeto de estudio. La primera década del presente siglo, marcó una discreta atención hacia los mismos; pero ninguna de las investigaciones tiene como centro a la programación dramatizada ${ }^{2}$. Resultan favorecidos los informativos, acompañados de la investigación sobre programas musicales, comunicación e identidad organizacional y estrategias de comunicación.

Si bien los emisores y, en este caso, la programación dramatizada ha despertado cierto interés en algunos investigadores, se debe precisar que las "miradas" no se han detenido en el proceso productivo en sí de dichos productos y, menos aún, en las mediaciones que se manifiestan en este proceso.

Sin embargo, esta situación es diferente en la programación informativa, incluyendo la radial, donde la producción de información y sus elementos mediadores sí han captado y captan el interés investigativo.

Considerando que producir un relato no es fruto de "un ejercicio de libertad de imaginación", sino que en este proceso ocurren "acotaciones comunicativas" y de otra índole, provocadas por determinadas acciones de los actores que intervienen en este proceso, se reitera el propósito de demostrar la validez de enfocar el análisis de la producción dramatizada radial desde la perspectiva de las mediaciones.

Al referirnos a la productora Radioarte, explicamos de forma general, cómo transcurre el proceso de creación de su programación variada, destacando los diferentes momentos que la misma incluye hasta la distribución del producto. Luego se analizan algunos aspectos, desde la perspectiva de las mediaciones, que se manifiestan en este proceso productivo.

2 A partir del 2005 ha existido un creciente interés en la Facultad de Comunicación Social de la Universidad de La Habana por el estudio de los emisores, específicamente con relación a los programas dramatizados, tales como los Trabajos de Diploma. Las novelas del aire. Panorámica del melodrama radial cubano de los años 40, de Nabil García Alfonso (2005); Lo que el río arrastra: Un acercamiento teórico-histórico a la radionovela cubana de la década del sesenta, de Sarahí García Contreras y Yeanny González Peña (2007); y, Cuando la felicidad llega al espacio. Tu Novela de Amor, de Anabel Quiñones (2012). 


\section{Desarkollo}

Radioarte, creada el 6 de abril de 1984, es la única productora de programas dramatizados que existe en Cuba. Junto a ella se encuentran emisoras que producen y transmiten programación dramatizada, pero solo esta entidad es la que se dedica a producir programas que envía a setenta y tres emisoras de todo el país.

La programación abarca seriados y unitarios organizados en diversos espacios: Novela Universal, Novela Cubana, Teatro, Cuento, Aventuras, Policíaco y otros programas de orientación social, de carácter histórico, jurídico y proyectos que pueden ser propuestos por escritores, instituciones, organismos o por la propia productora sobre los temas ya mencionados u otros de interés.

El enfoque de las mediaciones expuesto por Martín Serrano (1993) es válido para examinar y fundamentar, teóricamente, el proceso de producción de la productora ya mencionada.

Entre los aspectos analizados por el autor, que pudieran constituir pautas teóricas a considerar, se encuentran:

- Pertinencia del análisis de los procesos de producción comunicativa desde el enfoque de las mediaciones.

- El concepto de mediación. Su carácter institucionalizado.

- La conceptualización de mediación cognitiva y estructural.

- El producto comunicativo y sus dimensiones.

- Las tres mediaciones de la producción de comunicación pública.

\subsection{Pertinencia del análisis de los procesos de producción comunica- tiva desde el enfoque de las mediaciones.}

La comunicación producida desde lo dramatizado exige un carácter de proceso, donde todos sus momentos manifiesten un comportamiento orgánico, es decir, garanticen la dinámica de cada parte a su interior y, con ello, su interrelación con respecto al resto de las partes del proceso. Esta situación, por tanto, presupone la manifestación de ciertas mediaciones que intervienen en el devenir de estas acciones, imprimiéndole un carácter sistémico. 
Comprender la producción de este tipo de programación radial exige una visión dialéctica del mismo, en el cual las mediaciones tienen su papel.

Refiriéndose a la necesidad de este análisis, Martín Serrano señala que "La mediación pretende ofrecer un paradigma adecuado para estudiar todas aquellas prácticas, sean o no comunicativas, en las que la conciencia, las conductas $y$ los bienes entran en procesos de interdependencia (...)”3.

La necesidad de un enfoque basado en el análisis de la mediación se hace sentir cuando el manejo de la información, de los actos, de las materias, se manifiesta como una actividad que no puede ser disociada ni analizada por partes. La producción de información destinada a la comunicación pública es una de esas actividades (1993: 21).

Como ya expone Martín Serrano (1993) y se ha afirmado anteriormente, el proceso productivo de comunicación pública no puede analizarse por partes, sino en interrelación y asumiendo la conceptualización de la información sin reducirla a la programación informativa. Sobre la ampliación de este concepto se hace referencia más adelante.

Si se detiene el análisis en la creación de los programas dramatizados radiales en Radioarte, se observan cuatro momentos importantes: la escritura del libreto, su asesoría, grabación y distribución, cada uno de los cuales contiene en sí su propia dinámica que determina el comportamiento del proceso en su totalidad y permiten un resultado final, cuya calidad depende de cómo se desarrolle dicho proceso. El enfoque de las mediaciones es básico cuando se pretende construir, de forma general, el entretejido que se manifiesta en todos los aspectos que intervienen y hacen posible un programa dramatizado radial.

\subsubsection{Escritura del libreto}

Escribir el libreto es uno de los primeros momentos que marcan la producción de un programa radial, en el presente caso, del dramatizado. Se dice uno de los primeros momentos y no el primero, porque si bien tener el libreto en soporte de papel marca el final de una etapa y principio de otra,

3 Todas las cursivas son propias del autor citado.

Mediaciones Sociales, № 12, 2013, pp. 83-105. ISSN electrónico: 1989-0494.

DOI: http://dx.doi.org/10.5209/rev_MESO.2013.n12.45264 
antes de obtener el mismo, el escritor debe proponer un tema y analizarlo junto a la asesora y el director que correspondan.

Cuando se dice proponer, se indica que el tema escogido por el escritor debe, por supuesto, motivarlo a crear una obra, pero a la vez, corresponderse con la política de programación que rige en la institución, siendo este elemento uno de los que median los temas a tratar en el contenido de los mensajes, así como el modo de reflejarlos. En otras palabras, la política de programación es una mediación en los temas y enfoques que los escritores deben proponer para un programa dramatizado, "Tales narraciones ponen en relación los sucesos que ocurren con los fines y con las creencias en cuya preservación están interesados determinados grupos sociales" (Martín Serrano, 1993: 36).

Aprobado el tema a desarrollar se contrata al escritor y se establecen las tarifas de pagos, según características del libreto y del espacio al que corresponde la propuesta, o sea, si es aventura, radioteatro, radiocuento, policíaco, entre otros. Además, se aplica un aumento de pago por porciento, según criterio de la asesora por la complejidad del tema y calidad del escritor. Aquí intervienen mediaciones económicas que “imponen” los límites en las relaciones escritor-asesor.

Desde el punto de vista de la estructura formal, la creación de un libreto dramatizado también está mediada por otras exigencias económicas. En su página frontal debe reflejar el equipo de realización (el escritor solo indica las especialidades, es decir, asesora, director y grabador, entre otras, luego se escriben los nombres de los miembros del equipo de realización por especialidad). También se exponen los nombres de los personajes, cuya cantidad depende no solo de la dramaturgia, sino del tipo de programa (un espacio de orientación de quince minutos no debe tener más personajes que una novela de veinte minutos) y la clasificación de dichos personajes en protagónicos, co-protagónicos, actuaciones especiales y así sucesivamente, hasta clasificar a todos los actores y actrices para que reciban su pago según hayan sido clasificados.

Las competencias culturales también median el trabajo del escritor. No todos los escritores tienen la capacidad para escribir sobre todos los temas al no tener el mismo nivel de información sobre ellos, no manejar los términos correspondientes ni experimentar iguales motivaciones. 


\subsubsection{Asesoría del libreto}

Junto a la escritura del libreto se realiza su asesoría, pues por contrato el escritor debe entregar mensualmente una cantidad definida para ser asesorados e indicar el pago en dependencia de la calidad.

En el proceso de asesoramiento se analiza si el escritor cumplió con todos los pasos formales de presentación del libreto. Se realiza la lectura de reconocimiento y luego se hace el desmontaje para analizar la dramaturgia: planteamiento y desarrollo del conflicto, caracterización de los personajes, puntos de giros, progresión dramática, tratamiento del tema, personajes por capítulos hasta lograr el análisis integral de cada uno. Luego de hacer los arreglos propuestos, la asesora autoriza el pago del libreto, a través de los documentos previstos.

La asesoría requiere de especialistas con un alto nivel cultural que le permita hacer valoraciones, críticas, sugerencias, en fin, disímiles observaciones a un escritor. El trabajo de asesoría requiere de una diaria y constante observación de la vida cotidiana y conocer el contexto de otras épocas para velar por un adecuado reflejo en el libreto, por tanto, en esta etapa también están presentes las mediaciones económicas, políticas y culturales, pues la asesora tiene la función de velar que se cumplan las indicaciones de la política de programación, que a su vez, debe responder a los intereses gubernamentales y partidistas del país.

Las mediaciones económicas están presentes, ya que la asesora debe exigir que el libreto cumpla los requisitos que permitan no encarecer los capítulos, en caso de los seriados, ni el unitario, cuando se trate de estos. De hecho, la asesora debe conocer el presupuesto asignado al espacio para el que trabaja y distribuirlo según la cantidad de escritores con los que cuenta para lograr balance de oportunidades para los mismos, así como en los temas y estilos.

\subsubsection{Grabación del libreto}

Para iniciar este momento, el director o directora asignada para grabar el libreto, debe dar su aprobación, pues puede ocurrir que la asesoría no se realice con la calidad requerida, por diversas razones, haciendo que el director no apruebe el libreto para ser grabado y se devuelva a la asesora para enmendarlo junto al escritor. 
En el recorrido natural del proceso de producción, cuando el director acepta el libreto entregado, debe estudiarlo y realizar su montaje. Junto al resto del equipo de realización: grabador u operador de sonido, efectista y musicalizador, el director va definiendo efectos, música, planos, entre otros aspectos, según su punto de vista y respetando en lo esencial la idea expuesta por el escritor.

El modo en que transcurre este último aspecto de los cambios que el director realiza en el libreto, depende en gran medida, de las relaciones de trabajo que se establecen entre el escritor, asesor y director. En ocasiones el escritor le da total libertad al director para que realice los cambios que entienda y, en otras, debe consultársele al autor cada detalle a transformar en lo escrito originalmente.

La grabación de un programa dramatizado también está determinada por las mediaciones que ya se han mencionado. El director es el encargado de definir, finalmente, qué personaje será interpretado por un actor o actriz y si se pagará como actuación especial, de reparto, protagónico o coprotagónico y así sucesivamente, en dependencia de las características del libreto. Aquí también entran en juego las relaciones entre la asesora y el director, pues cuando son diáfanas, la primera es también consultada para este trabajo y se hace de conjunto.

Ya se había mencionado la responsabilidad del director en la clasificación del reparto y es aquí, precisamente, donde se manifiesta con claridad la presencia de mediaciones económicas, pues este proceso determina, en buena medida, el presupuesto que se invertirá en el programa, por tanto, además de lo económico intervienen las disposiciones establecidas por la productora que, sin dañar el aspecto artístico en cuanto a la dramaturgia, exigen el cumplimiento de estas disposiciones para emplear adecuadamente el presupuesto asignado a la entidad.

La mayor responsabilidad en el resultado final de un producto comunicativo es del director, el cual ejercerá su función con mayor o menor calidad, en dependencia de su nivel cultural, sensibilidad artística, conocimiento del lenguaje radial, por solo mencionar algunas de estas exigencias. Un director que tenga estas características y las cultive diariamente, posee las herramientas básicas para garantizar la calidad requerida en el programa unida al dominio de la técnica radial. 
Igual sucede con el resto de las especialidades que intervienen en el proceso. Un musicalizador debe conocer no solo de música, sino el contexto histórico del surgimiento y desarrollo de cada género para saber cuál corresponde a cada programa y cómo incorporarla a partir de su conocimiento del lenguaje radial.

Efectistas y grabadores no se excluyen de esta necesidad de conocimientos, si de verdad quiere lograrse un producto con calidad, pues los sonidos, ambientes y efectos no son iguales en todas las épocas históricas. Además, la calidad del efecto depende también del nivel artístico con que lo realice el efectista para lograr los sonidos lo más cerca posible a la realidad.

Todas estas especialidades están mediadas no solo por las competencias culturales, sino también por lo económico, pues su retribución depende de la clasificación del programa en Complejo y Muy Complejo, según definición de los especialistas en Programación de la Dirección Nacional de la Radio Cubana y que se refrenda en los documentos que rigen la política del Instituto.

Luego de grabarse el programa, este es analizado por una Comisión de Calidad que vela por el cumplimiento de los parámetros artísticos e institucionales que median este proceso de producción y que, en sentido general, se pueden agrupar en económicos, políticos y culturales.

\subsubsection{Distribución de los programas}

Al referirse a este momento del proceso productivo, Martín Barbero plantea que las estrategias que se aplican en este sentido

“(...) no son algo que se añada «después» para vender el producto, sino algo que ha puesto sus huellas en la estructura del formato bien sea en la forma que toma el corte narrativo (...) en los ingredientes diferenciales que introduce la diversificación de lo que sólo se verá «dentro» de un país o también fuera de él” (Martín Barbero, 1987: 239).

Si bien Martín Barbero se refiere a la producción televisiva, lo que señala es válido para el medio radial. También debe precisarse que este autor denomina este momento como "estrategias de comercialización". No se asume este término, porque Radioarte, hasta el momento, distribuye sus programas, pero no los comercializa, o sea, no son vendidos a las emisoras, sino que son distribuidos en discos compactos. 
Al igual que refiere Martín Barbero, las estrategias de distribución "no son algo que se añada «después»", estas se tienen en cuenta desde que se concibe el libreto, pues Radioarte debe satisfacer las exigencias de los radioyentes comprendidos en la cobertura de cada emisora que recibe sus programas. Por ello no todas los emiten por igual, sino que unas trasmiten Novela Universal, Radioteatro, Policíaco, es decir, cada una asume diferentes espacios de esta productora.

La distribución está mediada por las condiciones tecnológicas que posee la productora, pues se debe contar con la suficiente cantidad de discos compactos para reproducir las copias a enviar, computadoras para realizar este proceso, transporte para enviarlos. A su vez, en los pasos previos en la producción, los estudios deben contar con la tecnología digital para grabar el libreto, transportarlo al servidor y de allí al Departamento de Distribución, es decir, es una cadena donde la tecnología está en todo el proceso para garantizar su distribución.

Junto a la tecnología, los intereses institucionales median esta distribución. En ocasiones se priorizan los programas que contienen temas cuyo comportamiento social asumen determinadas características en un sector de la población ubicado en un área geográfica específica y a través de los mensajes radiales se orienta y educa a esa audiencia. Por ejemplo, VIH/SIDA, drogas, entre otros temas, pues las estrategias de distribución ponen "sus huellas en la estructura del formato", al definirse institucionalmente para qué espacio es más adecuado el tratamiento del tema y, en dependencia de ello, su "corte narrativo", así como la emisora en la que debe radiarse.

Es la distribución, desde la posición de emisores, la fase final del proceso productivo que permite la conjugación y expresión de las dimensiones del producto comunicativo, las cuales serán analizadas en otro momento del trabajo.

\subsection{El concepto de mediación. Su carácter institucionalizado}

Lo explicado anteriormente, en sentido general, sobre cómo transcurre el proceso de producción de un programa dramatizado en el contexto aquí analizado, revela la manifestación de mediaciones que intervienen en dicho proceso por cuenta de los actores que lo desarrollan, poniendo de manifiesto que 
"Favorecidos por la necesidad de una destreza técnica en la adquisición y elaboración de la información y en la distribución del producto, Mediadores e instituciones mediadoras monopolizan la función emisora, tanto cuando llevan a cabo su trabajo en el marco de la propiedad privada de los MCM, como cuando los realizan en régimen de propiedad estatal” (Martín Serrano, 1993: 94).

Esta función mediadora hace que las mediaciones se manifiesten en este proceso para imponer "límites a lo que puede ser dicho, y a las maneras de decirlo, por medio de un sistema de orden" (Martín Serrano, 2008: 76). Se trata de una productora estatal, en cuyo funcionamiento están presentes mediaciones económicas, políticas y culturales, tal como se muestra en la Figura 1, que van guiando el proceso creativo y establecen quiénes, cómo, cuándo y dónde deben hacer lo que les corresponde en cada momento de la producción, demostrando que estas mediaciones tienen un carácter institucionalizado, como lo señala Martín Serrano, y que no es ajeno a la realidad cubana:

"La característica de la información que se obtiene por conducto comunicativo radica en que ha sido seleccionada y preelaborada por otro u otros Actores diferentes del usuario. Por esa razón la consonancia entre el acontecer y el conocer que quepa atribuir al efecto de la comunicación está mediada por Alter; y cuando el mediador actúa a través de los MCM, que son instituciones sociales, la mediación está institucionalizada” (Martín Serrano, 1993: 14).

Figura 1. Mediaciones en el proceso de producción de programas dramatizados radiales

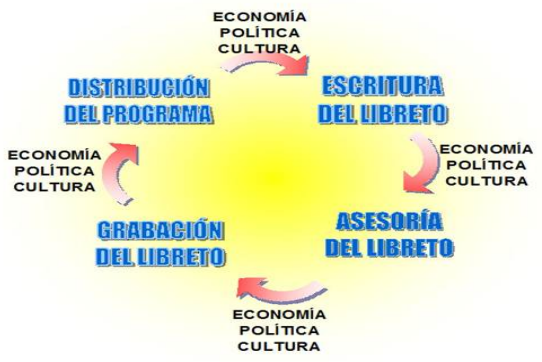

Fuente: elaboración propia. 
Es eso y no otra cosa lo que se hace en la producción de dramatizados: seleccionar y pre elaborar el contenido de los libretos, atendiendo a los diversos intereses y motivaciones de las audiencias, pero en los límites establecidos por las políticas de programación que, a su vez, debe ser coherente con la partidista y gubernamental del país. A ello se suma, como ha sido ya señalado en otro momento del trabajo, las características personales de los realizadores que también median estos procesos.

Más adelante, al hacer referencia a las tres mediaciones de la comunicación pública que señala Martín Serrano, se amplía sobre este proceso mediador institucionalizado que selecciona y pre elabora la información que será transmitida a la audiencia.

\subsection{La conceptualización de mediación cognitiva y estructural}

Reflejar la realidad social en la que está inmersa la audiencia y hacerlo a través del lenguaje y soporte material del medio de comunicación, es una "tarea agónica" propia de la vida diaria en el interior de los medios de comunicación. Estas acciones están dentro de los límites de lo que Martín Serrano reconoce como mediaciones cognitivas y estructurales. Las primeras se definen a partir de la relación entre el acontecer y la reproducción de normas sociales:

"El conflicto entre el cambio del acontecer y la reproducción de las normas sociales reclama una mediación cognitiva. La mediación cognitiva está orientada a lograr que aquello que cambia tenga un lugar en la concepción del mundo de las audiencias. Para proporcionarle dicho lugar a lo que en el entorno es nuevo o distinto, en ocasiones será preciso que la mediación cognitiva llegue a transformar esa concepción del mundo" (Martín Serrano, 1993: 135).

Mientras que la mediación estructural

“(...) está destinada a conseguir que aquello que irrumpe sirva para realimentar las modalidades comunicativas que cada medio adopta. Es una tarea agónica, en la que los artífices del medio se ven obligados a recurrir, para perpetuar la forma expresiva que producen, a aquella misma fuente de información que cada día vulnera los marcos formales de la comunicación; es decir, el acontecer imprevisible" (Martín Serrano, 1993: 135). 
La mediación cognitiva se relaciona con la representación del mundo que hacen los medios en los relatos, por tanto, incluye lo mítico al reiterar datos que son conocidos por la audiencia y que las conforta al establecer contacto con el "creer".

La mediación estructural está relacionada con los soportes de los medios de comunicación que determinan los modelos de producción asumiendo lo ritual, pues asegura "la repetición de formas estables del relato", dándole a la comunicación su carácter institucionalizado a través de los mediadores, o sea, esta mediación se relaciona con el "prever".

Tomando en consideración estas concepciones de Martín Serrano, los mensajes de los medios de comunicación, en su contenido, deben representar visiones del mundo que estén acorde a lo aceptado en la realidad de las audiencias y, por otro lado, deben ser ubicados dentro del modelo de producción previsto ya por el medio para que no acontezca el caos y se mantenga el orden defendido por la organización mediática. Así lo refiere el autor:

"En consecuencia, todos los medios se enfrentan con la tarea de encajar la novedad en el diseño material y conceptual que les caracteriza. Por eso los profesionales de los MCM median al propio medio de comunicación; utilizan el espacio y el tiempo disponibles para lograr un ajuste entre dos dimensiones: por una parte, la dimensión histórica del «acontecer»-que cuanto más imprevisto, más presiona para que se dé noticia de su emergencia- y, por otra, la dimensión práctica del «prever»-que presiona para que la confección se atenga a un programa invariante y tanto más estable cuanto más complejo sea el instrumento de comunicación-" (Martín Serrano, 1993: 144).

La producción de los dramatizados radiales se basa en conocimientos ya establecidos y reconocidos en la cultura e ideología profesionales de los actores que intervienen en el proceso. Se conoce y debe respetarse, en lo esencial, cómo escribir un libreto de una novela cubana, aventura, seriado histórico y así sucesivamente en cada espacio donde se "producen los mitos" y los "rituales".

La mediación cognitiva posibilita que el contenido del mensaje dramático no provoque disonancia ${ }^{4}$ en el radioescucha y consolide sus creen-

4 "La disonancia ha sido descrita por Festinger (1957) como un estado de incomodidad o tensión producido por la concurrencia de dos elementos cognoscitivos, igualmente aceptados 
cias respecto al tema que se le propone. Por su parte, la mediación estructural garantiza que dicho contenido se estructure dentro de las "formas estables del relato", ya probadas en la realización y que hacen posible el reconocimiento de un tema dentro de cada espacio como Radiocuento, Novela Biográfica, Novela Universal, por solo mencionar algunos, pero siempre de forma dramatizada ${ }^{5}$.

Además de las mediaciones cognitivas y estructurales, es factible el análisis de este proceso productivo, a partir de la concepción de Martín Serrano sobre los productos comunicativos y sus dimensiones.

\subsection{El producto comunicativo y sus dimensiones}

Desde el punto de vista de sus características narrativas los programas dramatizados ${ }^{6}$

"son aquellos programas producidos en forma escenificada, unitarios o de continuidad; originales o adaptados para la radio, que narran mediante personajes un conflicto, creando ambientes y escenarios sonoros y emplean todos los elementos que componen el sonido del mundo radiofónico. Recrean hechos reales o de ficción" (VV.AA, 2004: 22).

Si se define como producto comunicativo es "(...) un objeto fabricado que tiene un valor de uso concreto: poner la información que han elaborado unos sujetos sociales a disposición de otros” (Martín Serrano, 1993: 48).

En este sentido Thompson (2008: 186) se refiere a formas simbólicas, las cuales define como un "(...) amplio campo de fenómenos significativos, desde las acciones, gestos y rituales, hasta los enunciados, los textos, los

por el sujeto, que lleva a los sujetos afectados a realizar algún esfuerzo por restablecer la consonancia" (Martín Serrano, 2008: 71).

5 En algunas ocasiones se ha hablado en la productora, por parte de su Dirección, sobre la posibilidad de producir programas con otros modelos productivos y ha existido cierta resistencia pues implicaría cambios en el objeto social de la entidad y que se define como sigue: “... es la productora de programas dramatizados de radio, forma parte del Subsistema de la Radio Cubana en el ICRT, distribuye sin costo alguno los programas por las diferentes emisoras".

6 En el Manual del Control de la Calidad de la Programación Radial se emplea el término "programas escenificados", en el presente trabajo se asume "programas dramatizados" porque es el utilizado por todos los que intervienen en su producción. No obstante, la definición expuesta en el Manual no contradice el significado de ninguno de los dos términos. 
programas de televisión y las obras de arte” y le atribuye las siguientes características o aspectos:

- Intencional: presentar a los objetos como formas simbólicas es convertirlos en "fenómenos significativos", por lo que un sujeto los produce y emplea con determinada intención.

"Decir que un objeto fue producido, o que se percibe como si hubiera sido producido, por un sujeto capaz de actuar de manera intencional no significa, sin embargo, que el sujeto haya producido el objeto de manera intencional, o que éste sea lo que el sujeto tenía la intención de producir; significa simplemente que el objeto fue producido, o es percibido como si hubiera sido producido, por un sujeto acerca del cual podríamos decir, alguna vez, que lo hizo de manera intencional" (Thompson, 2008: 188).

Por supuesto que el significado de una forma simbólica no depende solo de la intención del sujeto que la produce, sino de diversos factores.

- Convencional: “(...) la producción, la construcción o el empleo de las formas simbólicas, así como su interpretación por parte de los sujetos que las reciben, son procesos que implican típicamente la aplicación de reglas, códigos o convenciones de diversos tipos" (Thompson, 2008: 189). La interpretación de las formas simbólicas se relaciona con las convenciones sociales en las que se construyen, es decir, con los códigos convencionales que hacen que estas formas se interpreten de uno u otro modo.

- Estructural: "las formas simbólicas son construcciones que presentan una estructura articulada” (Thompson, 2008: 191). Según el lenguaje del medio así será la estructura que adopta la forma simbólica.

- Referencial: “(...) las formas simbólicas son construcciones que típicamente representan algo, se refieren a algo, dicen algo acerca de algo" (Thompson, 2008:194). Estas formas simbólicas se refieren a los objetos de la realidad o creaciones imaginarias del sujeto, pero siempre tienen un referente sobre el cual expresarse.

- Contextual: “(...) las formas simbólicas se insertan siempre en contextos y procesos socio históricos específicos dentro de los cuales, y por medio de los cuales, se producen y reciben” (Thompson, 2008: 196). 
Sintetizando, se puede plantear que en Thompson estas características asumen lo que en Martín Serrano (1993) se plantea como la dimensión cognitiva del producto comunicativo, pues "está expresamente confeccionado para ofrecer información”. Asimismo, la definición de producto comunicativo no entra en contradicción con la de formas simbólicas, ambos conceptos son útiles al analizar el proceso de producción de comunicación, su utilización depende del enfoque que se asuma en el estudio.

La dimensión objetal del producto comunicativo para Martín Serrano,

“(..) se manifiesta, en primer lugar, porque se requiere un equipamiento y una organización (máquinas, especialistas de diferentes competencias) para la elaboración y distribución del producto comunicativo. En segundo lugar, porque se necesita un soporte material para que pueda ser difundido (periódicos, películas, cintas magnéticas, etc.)" (Martín Serrano, 1993: 125).

En Radioarte, ambas dimensiones se manifiestan cuando se analizan sus programas como transmisores de información a través de sus contenidos y al valorar el comportamiento de la tecnología para la producción y organización de todo este proceso.

La dimensión cognitiva se pudiera encontrar en la base de la concepción de las Funciones de los programas de radio, contenidas en su Manual de Calidad (2004: 20), en el cual se expone que "La Radio Cubana cumple como medio de Comunicación seis funciones que deben estar presentes en cada uno de los espacios".

- Educativa: contribuye al desarrollo de la capacidad intelectual del hombre, en el contexto de las necesidades de instrucción que demanda la sociedad, a la vez que trabaja en el aspecto gnoseológico de los problemas. Se puede apoyar en métodos e instrumentos didácticos y pedagógicos.

- Informativa: proporciona con sentido selectivo, los elementos básicos explicativos para difundir hechos, acontecimientos, teorías y previsiones de todas las esferas de la vida, ofrecidos a través de los géneros periodísticos.

- Cultural: estimula al desarrollo de valores no conocidos y reafirma el conocimiento de otros, en relación con los logros y aspiraciones espirituales del hombre. A su vez promueve y sirve de acicate creativo a los 
que descubren nuevos horizontes, fórmulas o técnicas no conocidas y llegan a encontrar placer o satisfacción en las ciencias, las artes o la literatura, incrementando el acervo cultural del ser humano.

- Recreativa: contribuye a complementar parte del tiempo libre de la población a través del esparcimiento, la distracción, la diversión, el entretenimiento y el recreo. Puede a su vez contribuir al disfrute estético.

- Orientación: contribuye a crear en el hombre un sistema de valores, actitudes y modelos de conducta positivos en consecuencia con los principios de la sociedad, a la vez que a la preservación y reafirmación de ellos. Logra además llevara la conciencia del ser humano patrones de conducta, hábitos y costumbres.

- Divulgativa: difunde actividades de todo tipo de instituciones de las diferentes esferas del quehacer humano y da a conocer otros aspectos de la vida cotidiana que resultan útiles a la población. Asimismo contribuye a propagar usos, hábitos, costumbres y consumo.

En las citas anteriores se observa cómo los programas dramatizados son productos comunicativos, formas simbólicas, que deben contener información que les permitan cumplir con las funciones ya referidas. Si se habla, por ejemplo, de orientación, habría que mencionar al programa ¿Qué piensa usted?, el cual está dirigido a orientar a la audiencia con relación a la conducta que se debe seguir ante determinadas problemáticas que se plantean y termina haciendo un llamado al oyente a pensar en el tema.

A continuación se muestra la escena final de uno de estos programas, que ilustra las características del mismo, mencionadas anteriormente. 
Tabla 1. Escena final de uno de estos programas que ilustra sus características

\begin{tabular}{|c|c|}
\hline SONIDO & $\begin{array}{l}\text { TRANSICION MUSICAL LIGA AMBIENTE PREUNIVERSI- } \\
\text { TARIO }\end{array}$ \\
\hline IBIS & Tienes que cambiar esa cara, Arelis. Así no puedes seguir. \\
\hline AREL & $\begin{array}{l}\text { (ARREPENTIDA) Ay, si yo pudiera echar el tiempo hacia atrás. Si } \\
\text { yo pudiera volver a ese campismo y no haberle mirado ni la cara al } \\
\text { tal Andel ese. (P) Dios mío no debí haberle hecho eso a Alfre, él no } \\
\text { se lo merecía y yo lo quiero tanto. (SUSPIRA) Terminé engañándo- } \\
\text { lo y pegándole una enfermedad de transmisión sexual. No tengo } \\
\text { cara para volver a mirarlo, y mucho menos a sus padres. Quisiera } \\
\text { tener alas y salir volando para otro lugar, pero no soy una mariposa, } \\
\text { y esto es lo que me toca, enfrentarlo. }\end{array}$ \\
\hline IBIS & Y pensar que pudo ser peor la cosa. Bien pudiste coger un SIDA. \\
\hline AREL & $\begin{array}{l}\text { Cada vez que pienso en eso, siento escalofríos. Yo que te criticaba a } \\
\text { ti, por tanta protección en tus relaciones sexuales. Si me hubiera } \\
\text { protegido no estaría pasando por esta situación. De ahora en adelan- } \\
\text { te tendré mucho cuidado en lo que haga, de eso puedes estar segura. }\end{array}$ \\
\hline IBIS & $\begin{array}{l}\text { Sí, Arelis, de ahora en adelante, debes tener mucha precaución. (P) } \\
\text { Mira, lo que yo te dije es cierto. El preservativo no impide que } \\
\text { disfrutes el sexo. Todo está en tu cabecita. Si no le pones coco al } \\
\text { asunto, ni te acuerdas que tu pareja lo tiene puesto. Evitas enfermar- } \\
\text { te, y también un embarazo, que no nos conviene a ninguna de las } \\
\text { dos en estos momentos. (TR) ¿Pudieron localizar a Andel? }\end{array}$ \\
\hline AREL & $\begin{array}{l}\text { (SUSPIRA) Ojalá, así le pondrían tratamiento y no contagiaría a } \\
\text { otras personas. }\end{array}$ \\
\hline IBIS & $\begin{array}{l}\text { Pero eso es trabajo de la enfermera encuestadora, ella tiene que } \\
\text { hacer lo posible por cortar la cadena de transmisión de la gonorrea. }\end{array}$ \\
\hline AREL & Sí, yo lo sé Ibis, pero se fue. \\
\hline IBIS & ¿Cómo que se fue, mija? \\
\hline AREL & Oye, que se fue del país. El papá lo había reclamado. \\
\hline IBIS & $\begin{array}{l}\text { Ay, mi madre, que cosa más grande. (TR) Bueno, ahora ya aprendis- } \\
\text { te la lección, pero la vida sigue. El fin de semana te quiero en mi } \\
\text { casa, tenemos casi arriba las pruebas de ingreso y hay que ponerse } \\
\text { para las cosas. }\end{array}$ \\
\hline AREL & $\begin{array}{l}\text { Claro, tienes razón. Aprendí la lección, y nada, la vida continúa } \\
\text { como tú bien dices. (TR) ¿Qué piensa usted? }\end{array}$ \\
\hline SONIDO & $\begin{array}{l}\text { LIGA TEMA DESPEDIDA BREVE BAJA A FONDO MANTIE- } \\
\text { NE }\end{array}$ \\
\hline LOC & CRÉDITOS \\
\hline SONIDO & SUBE A FIN \\
\hline
\end{tabular}

Aquí se expresa la dimensión cognitiva, pues se informa a la audiencia, en este caso a los jóvenes, la necesidad de protección para evitar las infecciones de transmisión sexual. 
La dimensión objetal está presente cuando en el análisis se destaca la necesaria presencia de los actores que intervienen en la creación de este producto, es decir, el escritor, asesor, director y las otras especialidades que participan en la producción del programa, incluyendo quienes lo distribuyen. Además, aquí se tiene en cuenta el soporte material (los discos compactos) a través de los cuales se hace entrega a las emisoras de lo producido.

Por ello, tomando en consideración las dos dimensiones antes mencionadas, se considera que "La comunicación pública provee a los miembros de la comunidad de relatos (orales, escritos, mediante imágenes) en los que se les propone una interpretación del entorno (material, social, ideal) y de lo que en él acontece" (Martín Serrano, 1993: 36).

\subsection{Las tres mediaciones de la producción de comunicación pública}

Cuando en la literatura se habla sobre la producción informativa, refiriéndose solo al área periodística, la mayoría de los autores de una forma $u$ otra reconocen tres etapas en el proceso de producción noticiosa: Recolección de la información, Selección del material informativo y Presentación de la información.

Estas etapas ya son referidas por Martín Serrano como mediaciones cuando expresa:

“(...) las tres mediaciones que se le encomiendan a la producción de comunicación pública: la selección de unos u otros aconteceres de referencia; la elección de unos u otros datos a propósito de esos aconteceres y la vinculación de tales datos del acontecer con otros evaluativos; la presentación del relato que describe lo que acontece en una u otra forma expresiva" (Martín Serrano, 1993: 106).

En la creación del dramatizado, al igual que en la producción informativa se determina qué va a dramatizarse, se busca y recoge la información y luego se procesa y se configura en cada género según las características del tema a tratar. La diferencia está en la especialización de los sujetos que cumplen esas tareas y las condiciones específicas para hacerlas?

7 Cfr. Fonseca, B. (2011): "Las rutinas productivas en la programación dramatizada de $R a$ dioarte", Revista Razón y palabra, No.77, agosto-octubre. Disponible en: www.razonypalabra.org.mx 
Es por ello la identificación de los programas según el tipo de relato que asumen. La información seleccionada no es igual para todos los espacios, así como su presentación. Todos son dramatizados, pero en el contenido difieren, pues un programa histórico no posee las mismas características de una aventura o radioteatro.

La información para una Novela Cubana no se procesa del mismo modo que se haría para la Novela Universal, pues la primera se basa en la vida cotidiana de la sociedad en el contexto cubano y la segunda refleja lo mejor de la literatura universal.

Aquí información se asume en el mismo sentido que Martín Serrano, quien la considera "En el sentido técnico del término «información» que significa precisamente la pérdida de variedad de un repertorio de sucesos y la disminución del número de formas alternativas que permitirían presentar los sucesos" (Martín Serrano, 1993: 108).

El autor destaca la imposibilidad de no perder información desde el instante que ocurren los hechos hasta el momento que son presentados a las audiencias. Por muy exhaustiva que sea la investigación realizada sobre un tema siempre habrá "pérdida de información" cuando se reelabora para un programa. Además, no puede evitarse que el sujeto deje su impronta en el objeto investigado y en el producto creado, es decir, no se puede "descentrar al sujeto que elabora la información, respecto del producto comunicativo que realiza".

Esta situación es inevitable en el proceso productivo de Radioarte. Si se detiene el análisis en un Radioteatro referido a la sociedad cubana actual, en él se encontrará una "disminución del número de formas alternativas que permitirían presentar los sucesos", pues se hace abstracción de un fragmento de la realidad, el cual a su vez, es fragmentado para su presentación.

Algo semejante sucede cuando en la Novela Universal se radian temas, por ejemplo, relacionados con el siglo XVIII en un país de América Latina o Europa, donde las fuentes de información son documentales y dicha realidad es más alejada de la actual.

Lo aquí planteado no se relaciona con la veracidad o falsedad de lo dramatizado, sino que el sujeto cognoscente está limitado por sus condiciones históricas sociales, en primer lugar, para reflejar el mundo que le 
rodea en su totalidad. Por otra parte, necesariamente debe hacer abstracción de la parte de esa realidad a la que presta atención, en este caso, la que representa en forma de dramas en los programas radiales, pues humanamente también está limitado al exponer esos mensajes de dicha realidad.

\section{CONCLUSIONES}

Desde su surgimiento y a lo largo de la historia de la radio, su magia ha cautivado a miles de millones de radioyentes que han sido y son atrapados por los programas informativos que nos actualizan con inmediatez de lo acontecido, la música que nos agrada escuchar o las radionovelas que nos atrapan capítulo a capítulo con la maldad o inocencia de los personajes que en ellas intervienen. Sin embargo, al menos en el contexto cubano, este medio no ha sido muy investigado, lo que está siendo revertido, discretamente, en las últimas décadas.

Si analizamos los temas que han despertado interés, en ellos no se destacan el estudio de emisores, menos aún, referido al proceso productivo de los dramatizados radiales. Este momento productivo carece de investigaciones que lo fundamenten desde una perspectiva teórica, la cual puede enriquecerse, en gran medida, tomando en cuenta el enfoque de las mediaciones sistematizado por Martín Serrano.

Muchos de los planteamientos de este autor desde las mediaciones, pueden ser analizados en el proceso de producción de Radioarte, pues los programas dramatizados que aquí se elaboran atraviesan por diversas fases, las cuales deben comportarse de forma sistémica y dialéctica para garantizar el producto comunicativo final y, como parte de este proceso, se encuentran las mediaciones.

Durante la producción de un programa dramatizado radial se manifiestan diversas mediaciones, que parten de los roles que deben cumplir los miembros de esta productora, los cuales son mediadores que, desde lo económico, político y cultural, definen el cómo, cuándo, dónde y qué hacer en todos los momentos del proceso de producción, contribuyendo a la gestión de la comunicación que se desarrolla en esta entidad. 


\section{BIBLIOFRAFÍA}

FONSECA, BEATRIZ (2011): "Las rutinas productivas en la programación dramatizada de Radioarte”, Razón y palabra, $\mathrm{n}^{\mathrm{o}}$ 77, agosto-octubre. Disponible en: www.razonypalabra.org.mx.

García Alfonso, Nabil (2005): "Las novelas del aire. Panorámica del melodrama radial cubano de los años 40”. Trabajo de Diploma. Facultad de Comunicación Social, Universidad de La Habana.

García Contreras, Sarahí y González Peña, Yeanny (2007): “Lo que el río arrastra: Un acercamiento teórico-histórico a la radionovela cubana de la década del sesenta”. Trabajo de Diploma. Facultad de Comunicación Social, Universidad de La Habana.

MARTÍn BARBERO, Jesús (1987): De los medios a las mediaciones: comunicación, cultura y hegemonía. Barcelona: Gustavo Gili.

Martín Serrano, Manuel (1993): La producción social de la comunicación. Madrid: Alianza.

Martín Serrano, Manuel (2008): La mediación social. Madrid: Akal.

QUiÑones, AnABEL (2012): "Cuando la felicidad llega al espacio. Tu Novela de Amor”. Trabajo de Diploma. Facultad de Comunicación Social, Universidad de La Habana.

ThOMPSON, John B. (2008): Ideología y cultura moderna. Teoría crítica social en la era de la comunicación de masas. México: Universidad Autónoma Metropolitana, Unidad Xochimilco, división de Ciencias Sociales y Humanidades.

VV.AA (2004): Manual de Control de la Calidad de la Programación Radial. La Habana: ICRT, Dirección de Control y Planeamiento de la Radio Cubana. 


\section{PARA CITAR ESTE TRABAJO EN BIBLIOGRAFÍAS:}

BEATRIZ ELENA FONSECA MUÑOZ (2013): "La producción de programas dramatizados radiales: un análisis desde las mediaciones", Mediaciones Sociales. Revista de Ciencias Sociales y de la Comunicación, $\mathrm{n}^{\mathrm{o}}$ 12, pp. 83-105. DOI: http://dx.doi.org/10.5209/rev_MESO.2013.n12.45264

\section{La autora}

${ }^{(*)}$ Beatriz Elena Fonseca Muñoz, es Profesora Auxiliar de la Facultad de Comunicación Social de la Universidad de La Habana. Máster en Ciencias de la Comunicación. Profesora Adjunta del Centro de Estudios de la Radio y la Televisión y miembro de su Consejo Técnico Asesor. Asesora de programas dramatizados radiales. Ha impartido cursos de postgrados y diplomados sobre Teoría de la Comunicación y Propaganda. En la Universidad Bolivariana de Venezuela impartió el Módulo de Teoría de la Comunicación, en la Maestría de Comunicación de la Universidad de La Habana y participó en la Asesoría de Tesis de dicha Maestría. Ha sido consultante y oponente de tesis de maestría sobre temas de la radio. Ha participado en eventos, tales como los Encuentros Iberoamericanos de Radiodifusión organizados por la Radio Cubana y FELAFACS, 2009.

RECIBIDO: 02 de noviembre de 2012.

ACEPTADO: 05 de mayo de 2013. 